\title{
Dramatic Improvement of Segmental Zoster Paresis by Late-onset Antiviral Therapy
}

Halil Onder $M D^{1}$, Gokcen Celik $M D^{2}$ iD

${ }^{1}$ Department of Neurology, Yozgat City Hospital

${ }^{2}$ Department of Dermatology, Yozgat City Hospital

Date of submission: $26^{\text {th }}$ April 2021

Date of acceptance: $4^{\text {th }}$ November 2021

Date of publication: $1^{\text {st }}$ December 2021

\section{Abstract}

Focal paresis secondary to Herpes zoster (HZ) is a rare neurological complication and should be kept in mind in the differential diagnosis of acute weakness with corresponding dermatomal rashes. In this case report, we illustrate an 84-year-old male patient, who presented with focal upper extremity weakness following $\mathrm{HZ}$ on right C4-8 dermatome. After clinical and electrophysiological evaluations, segmental zoster paresis was diagnosed. Although antiviral medication (oral brivudine) had been administered previously, oral valacyclovir and low dosage methylprednisolone were initiated. In the following 2 months interval, a progressive and dramatic recovery in extremity weakness and neuropathic pain were achieved. The clinical course of this patient may give substantial perspectives regarding the clinical evaluation of similar cases.

Key words: Antiviral therapy, Herpes zoster, Improvement, Paresis.

\section{Introduction}

$\mathrm{H}$ erpes zoster (HZ) is caused by the reactivation of Varicella Zoster Virus (VZV) and commonly presents in the elderly. ${ }^{1}$ Rarely, it can manifest as segmental paresis that typically occurs 2 to 3 weeks after the herpetic rash and usually affects the myotome that corresponds to the dermatomal distribution. ${ }^{2}$ The prevalence of segmental motor involvement in $\mathrm{HZ}$ is estimated to be in rates of $0.5 \%$ to $5 \% .{ }^{3,4}$ However, most patients with $\mathrm{HZ}$ is affected from the regions including roots of the ophthalmic branch of the trigeminal nerve, upper cervical (C2-3), or thoracic nerve roots in which motor involvement may be strictly hard to be detected in the clinical practice. Therefore, the authors suggests that the true prevalence of this entity in

Access this article online
Website: https://www.nepjol.info/index.php/NJN
DOI: https://doi.org/10.3126/njn.v18i4.36631
HOW TO CITE
Onder H, Celik G. Dramatic Improvement of Segmental Zoster
Paresis by Late-onset Antiviral Therapy. NJNS. 2021;18(4):44-8.

\author{
Address for correspondence: \\ Dr Halil Onder, \\ Number 8, Erdogan Akdag Street \\ Yozgat City Hospital Center, \\ Yozgat- 66100, Turkey \\ E-mail: halilnder@yahoo.com \\ Phone: +900-5376836864
}

Copyright (C) 2021 Nepalese Society of Neurosurgeons (NESON)

ISSN: 1813-1948 (Print), 1813-1956 (Online)

This work is licensed under a Creative Commons Attribution-Non Commercial 4.0 International License.
HZ may be far more than supposed. ${ }^{4}$ In accordance with this thought, studies using electrophysiological studies (EPS) remark considerably higher rates of motor system disturbance in HZ. ${ }^{5,6}$ Although there are several reports illustrating samples of segmental zoster paresis, there are still many controversies regarding many aspects including pathophysiology, classification, diagnosis, and clinical evaluation of this devastating entity.

Herein, we present a case of $\mathrm{HZ}$ who developed severe paresis of the right upper extremity and was admitted at subacute phase of the condition. Although he had taken antiviral therapy previously in the acute period, the antiviral therapy was repeated which provided dramatic improvement in the paresis. Via the presentation of this patient and related literature data, we will discuss primarily the efficiency of late antiviral therapy in patients with similar conditions. Besides, we propose some pathophysiological explanations and discuss the rationale of classifying distinct cases of $\mathrm{HZ}$ with paresis.

\section{Case report}

An 84-year-old male patient with a history of diabetes mellitus, hypertension, and ischemic heart disease presented to our neurology clinic with motor weakness in his right upper extremity and neuropathic pain in right shoulder extending to the forearm and partly recovered vesicular rashes on the right $\mathrm{C} 4-8$ dermatomes which had emerged approximately 1.5 months ago (Figure 1).

At that time, the patient consulted with dermatology due to neuropathic pain and vesicular rashes where the diagnosis of herpes zoster was made and 1x $125 \mathrm{mg}$ oral brivudine was administered for 7 days. A mild- 


\section{Dramatic Improvement of Segmental Zoster Paresis}

moderate improvement in the vesicular rashes and slight improvement in neuropathic pain were achieved after completion of the therapy. However, the patient continued to suffer from pain in the following course, and additionally, right upper extremity weakness had developed progressively nearly two weeks after the clinical presentation. He had visited distinct polyclinics several times (including dermatology, physical therapy rehabilitation, orthopedics and traumatology) before admission to our clinic. However, no additional treatment was initiated. Vital signs at admission to our clinic were within normal limits. On physical examination, vesicular rashes on $\mathrm{C} 4-8$ dermatome which had begun to resolve were recognized. Neurological examination revealed that right biceps, triceps, and brachioradialis reflexes were absent. Motor examination showed marked weakness both in the proximal and distal extremities (proximal: 2/5, distal: $3 / 5$ on MRC). Sensory evaluations revealed slight hypoesthesia from $\mathrm{C} 4$ to $\mathrm{C} 8$ dermatomes. Complete blood count, erythrocyte sedimentation rate, biochemistry profile, and thyroid function test results were within normal. Serological tests revealed negative varicella zoster virus (VZV) IgM results was and positive result for VZV IgG. Magnetic resonance imaging (MRI) of the cervical spine and right shoulder joint showed moderate osteoarthritis of the right shoulder joint. However, there was no evidence of spinal disease to explain the paresis. EPS showed a slight reduction in the amplitudes of compound motor action potentials of median, ulnar and radial nerves, whereas sensory nerve action potentials of ulnar, median, medial, and lateral antebrachial cutaneous nerves were within normal limits. F wave latencies were mildly prolonged (Figure 2). Needle electromyography revealed evidence of extensive acute denervation including fibrillation and positive sharp waves, polyphasia and reduced recruitment in myotomes of the C4-8 (supraspinatus, deltoid, triceps, biceps, first dorsal interosseous, abductor digiti minimi, abductor pollicis brevis) prominently in the proximal muscles and also cervical paraspinal muscles.

Taken together, the diagnosis of herpes zoster and associated segmental zoster paresis was established and based on the findings of EPS, the pathology was classified as radiculopathy and/or anterior motor neuron affection. The patient was referred to the dermatology clinic. Although he had received therapy for herpes zoster 1.5 months ago, valacyclovir 3x1 gm was initiated over the 7 days and low dosage methylprednisolone $40 \mathrm{mg}$ daily was also initiated and administered over the following two weeks by tapering the dose. A significant recovery in paresis was achieved following initiation of treatment and such that at the follow-up visit after two months, the patient could use his hand nearly as the premorbid state (proximal muscle strength of right upper extremity: 4/5, distal muscle strength: $4+/ 5$ ).

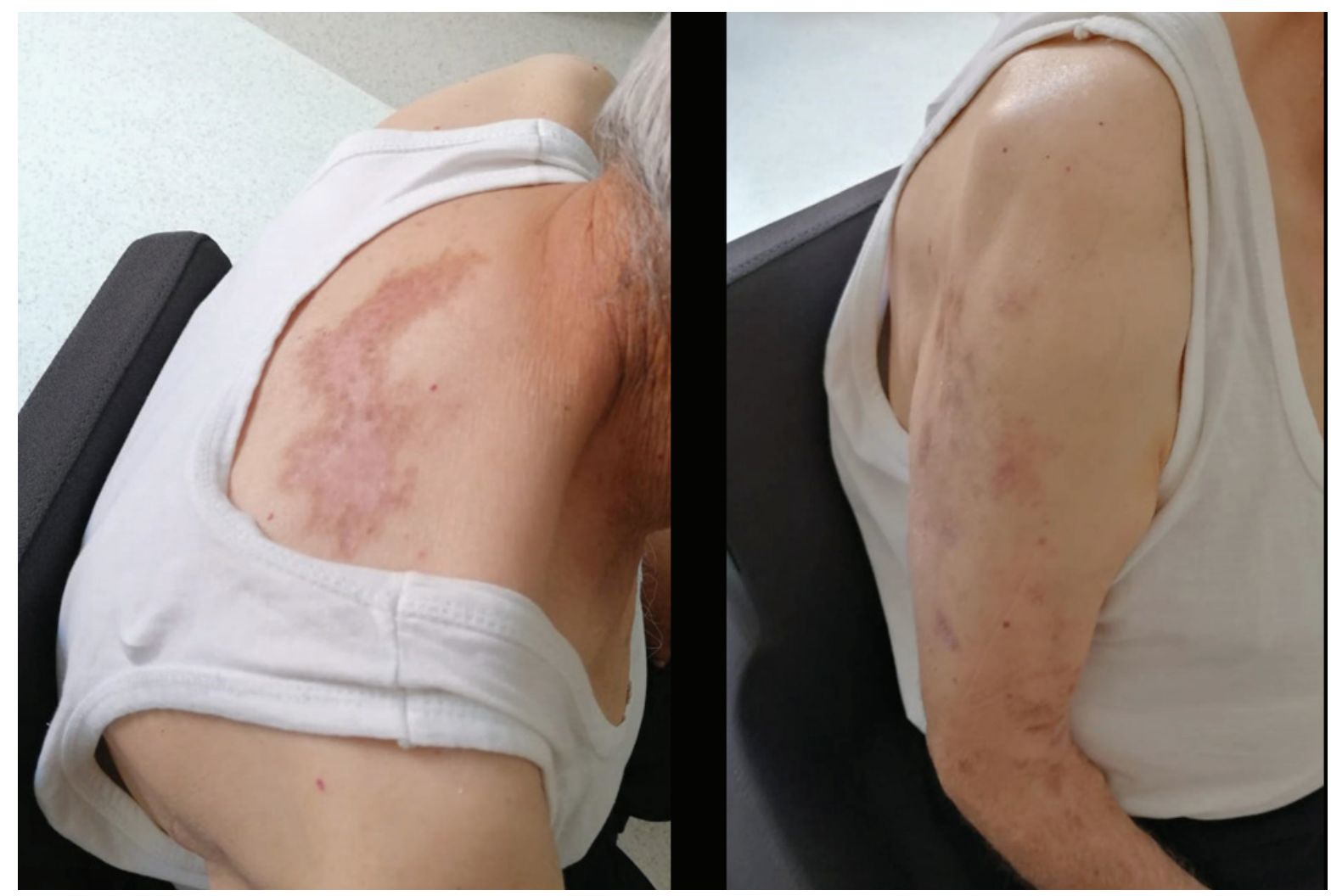

Figure 1: The images showing partly resolved vesicular rashes on C4-8 dermatomes. 


\section{Onder et al}

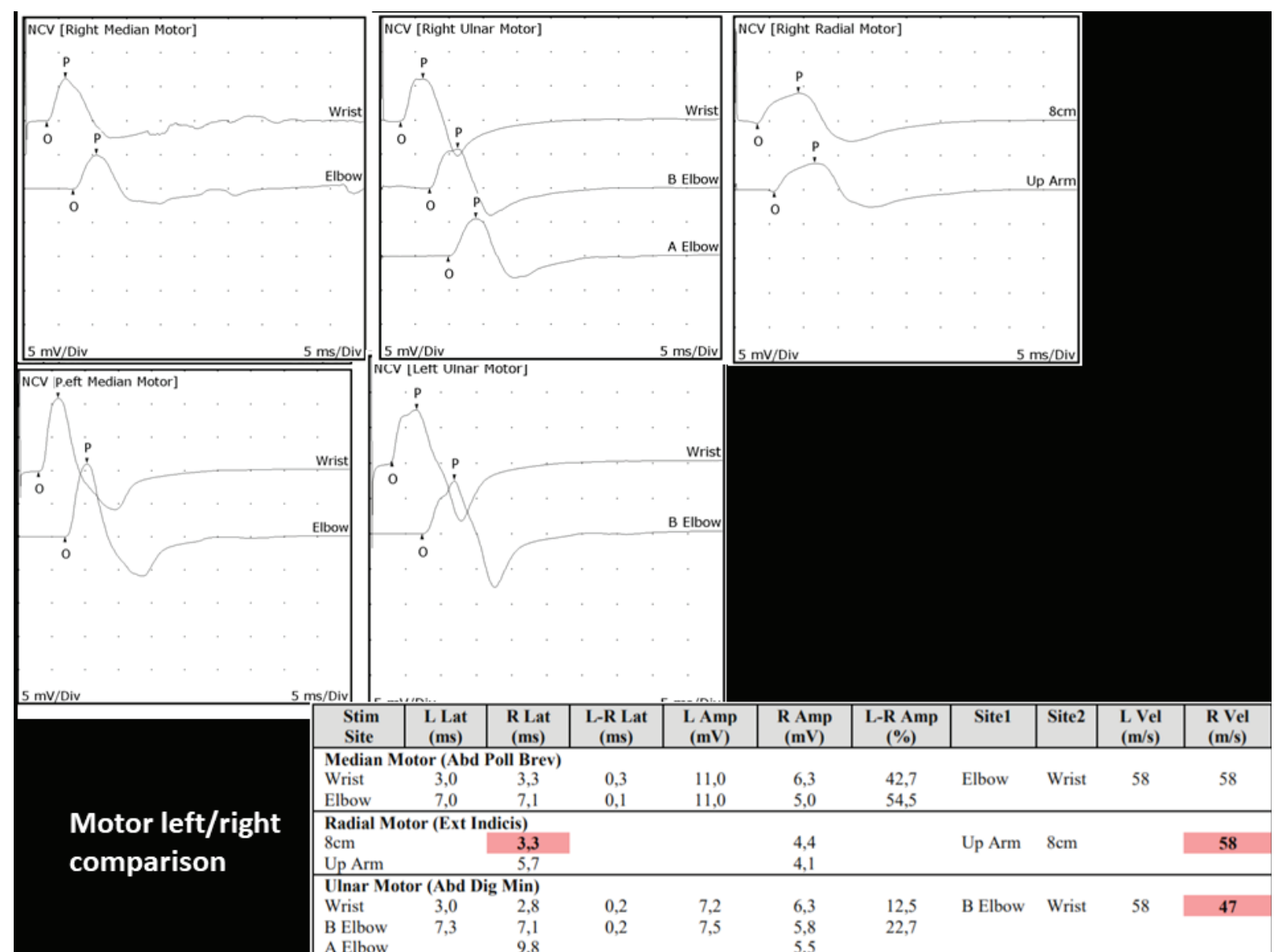

Figure 2: The images showing the comparative results of the motor nerve conduction studies. Slight reduction in the amplitudes of compound motor action potentials of right median, right ulnar and right radial nerves are detected.

\section{Discussion}

Although the exact pathogenesis of paresis associated with $\mathrm{HZ}$ is unknown, the association between the involved myotome and the dermatome of the rash leads to a suspicion of viral spread from the dorsal root ganglion to the anterior horn cells or anterior spinal nerve roots leading to inflammatory damage. ${ }^{7}$ The dysfunction of the motor nerve is hypothesized to occur via a mechanism of hypervascularity or disruption of the blood nerve barrier caused by inflammation. ${ }^{7}$ Radiculopathy is a common form of herpes zoster-related motor weakness ${ }^{7-9}$ which was also the case in our patient. However, more rarely, motor disturbance in the form of plexopathy has also been described in HZ. ${ }^{10,11}$ Previous reports remark that the improvement period is variable; ranging between 1 and 2 years $^{6,12}$ and some group of patients fail to improve completely which is explained by the death of motor neuron cells. ${ }^{13}$ In light of the literature data, we think that the dramatic response to antiviral treatment in our patient may constitute a critical point for further discussions.

Antiviral therapy is the gold standard therapy of HZ, however, classically antiviral for patients with uncomplicated herpes zoster is recommended within the first 72 hours of symptom onset. ${ }^{14}$ On the other hand, it has been suggested that antiviral therapy after 72 hours may be administered if new lesions are appearing at the time of presentation, as this indicates ongoing viral replication. ${ }^{14}$ The role of antiviral agents in the management of $\mathrm{HZ}$ with motor paresis has not been evaluated in a controlled fashion. Remarkably, although initiation of antiviral therapy is recommended in all immunocompromised patients with herpes zoster, even if they present after the acute period of manifestations, ${ }^{15}$ its indication in patients with motor deficits in the subacute period remains obscure. The remarkable points were that the patient admitted to our clinic 1.5 months after the clinical manifestations and he had received antiviral therapy previously at the onset of symptoms. However, after deliberations with the dermatologist, the administration of antiviral therapy with low dose corticosteroid therapy was decided which provided a dramatic improvement in paresis. In light of the limited literature regarding this issue, we think that our case drawing attention to the dramatic efficiency of antiviral therapy even at the subacute period in HZ with paresis may provide crucial perspectives. In the report by 


\section{Dramatic Improvement of Segmental Zoster Paresis}

Yoleri et al., a female patient with segmental zoster paresis of the upper extremity is presented at whom EPS supported a brachial plexopathy. ${ }^{8}$ Similar to our patient, their case was admitted to the clinic 6 weeks after appearance of the vesicular eruptions, and pain with paresis had developed two weeks after rashes. However, the authors had not initiated antiviral therapy (although antiviral therapy had not also been administered in the acute period) and only slight improvement could be achieved with physical therapy in the following two months period. In the other report of case series by Arslan et al. ${ }^{16}$ three patients with segmental limb paresis due to HZ associated plexopathy are illustrated. All the patients were admitted in the subacute period and antiviral therapy was initiated in all of these patients which provided only mild recovery of paresis in the subacute period. In a crucial report by Liu et al., ${ }^{6}$ the authors presented the detailed electrophysiological and clinical data of 8 cases with segmental zoster paresis which they classified as cases with preganglionic lesions $(n=2)$, cases with postganglionic lesions $(n=2)$ and those with combined pre- and postganglionic lesions $(n=4)$. Antiviral drugs were administered in all of the patients; however, the remarkable point may be that the most effective recovery in paresis was achieved in case 1 and case 8 who were electrophysiologically classified as cases with preganglionic lesions. ${ }^{6}$ In our case, all the sensory nerve examinations were within normal limits and paraspinal muscle needle examination also showed neurogenic denervation revealing the anatomical localization of the pathology as radiculopathy and/or anterior motor neuron affection that can also be evaluated as cases with preganglionic lesions. It is emphasized that the course of paresis in HZ may be positively affected by administering antiviral therapy, which is hypothesized to reduce peripheral sensory axonopathy and prevent viral spread. ${ }^{17}$ We hypothesize that the viral spread may lead to first anterior motor neurons and, then expand more distally resulting in plexopathy in the late phase which's response to antiviral therapy may be poorer. Taken together, we think that the patients with segmental zoster paresis with the subtype of preganglionic lesions (radiculopathy and/ or anterior motor neuron affection) may be more prone to have a favorable response to antiviral therapy. Therefore, there may be a rationale to more likely administer antiviral therapy in those patients with preganglionic lesions even after the acute period of the disease. However, this is only a unique case and many other individual factors other than antiviral therapy might have influenced the clinical output. For instance, we have also initiated low dose corticosteroid in addition to valacyclovir. There is a literature evidence supporting the use of corticosteroids in segmental zoster paresis, however, there is no a consensus regarding the dosage ${ }^{6,8}$. Corticosteroids, with strong antiinflammatory effects, may effectively improve the levels of inflammatory factors, reduce nerve injury, and promote the regression of blisters as well as improve nerve pain. ${ }^{18}$ Considering the possible worsening of glucose control, we preferred to initiate low dosage methylprednisolone. Therefore, we cannot distinguish the contributory effect of corticosteroid therapy in this favorable response.

\section{Conclusion}

We think that the administration of antiviral therapy in segmental zoster paresis even in the subacute period of the condition may provide substantial benefits in the management of patients. However, the advantage of lateonset antiviral therapy with or without steroid and the determination of the potential subgroup of patients those more likely to respond well to therapy constitute critical questions to be investigated. Future reports of multicenter large case series are warranted to clarify the debates regarding the treatment approaches in $\mathrm{HZ}$ patients with paresis with distinct clinical scenarios.

\section{Financial Disclosure: None}

Conflict of Interest: None

\section{References}

1. Yawn BP, Gilden D. The global epidemiology of herpes zoster. Neurology. 2013;81(10):928-930. https://doi.org/10.1212/WNL.0b013e3182a3516e

2. Kennedy PG, Barrass JD, Graham DI, Clements GB. Studies on the pathogenesis of neurological diseases associated with Varicella-Zoster virus. Neuropathol Appl Neurobiol. 1990;16(4):305-316. https://doi. org/10.1111/j.1365-2990.1990.tb01265.x

3. Gupta SK, Helal BH, Kiely P. The prognosis in zoster paralysis. J Bone Joint Surg Br. 1969;51(4):593-603. https://doi.org/10.1302/0301-620X.51B4.593

4. Thomas JE, Howard FM, Jr. Segmental zoster paresis-a disease profile. Neurology. 1972;22(5):459-466. https://doi.org/ 10.1212/wnl.22.5.459

5. Mondelli M, Romano C, Rossi S, Cioni R. Herpes zoster of the head and limbs: electroneuromyographic and clinical findings in 158 consecutive cases. Arch Phys Med Rehabil. 2002;83(9):1215-1221. https:// doi.org/10.1053/apmr.2002.33989

6. Liu Y, Wu BY, Ma ZS, et al. A retrospective case series of segmental zoster paresis of limbs: clinical, electrophysiological and imaging characteristics. BMC Neurol. 2018;18(1):121. https://doi. org/10.1186/s12883-018-1130-4

7. Hanakawa T, Hashimoto S, Kawamura J, Nakamura M, Suenaga T, Matsuo M. Magnetic resonance imaging in a patient with segmental zoster paresis. Neurology. 1997;49(2):631-632. https://doi. org/10.1212/wnl.49.2.631 


\section{Onder et al}

8. Yoleri O, Olmez N, Oztura I, Sengul I, Gunaydin $\mathrm{R}$, Memis A. Segmental zoster paresis of the upper extremity: a case report. Arch Phys Med Rehabil. 2005;86(7):1492-1494. https://doi.org/10.1016/j. apmr.2004.09.032

9. Braverman DL, Ku A, Nagler W. Herpes zoster polyradiculopathy. Arch Phys Med Rehabil. 1997;78(8):880-882. https://doi.org/ 10.1016/s00039993(97)90204-5

10. Alshekhlee A, Tay E, Buczek M, Shakir ZA, Katirji B. Herpes zoster with motor involvement: discordance between the distribution of skin rash and localization of peripheral nervous system dysfunction. J Clin Neuromuscul Dis. 2011;12(3):153-157. https://doi. org/10.1097/CND.0b013e31820d4f31

11. Choi JY, Kang CH, Kim BJ, Park KW, Yu SW. Brachial plexopathy following herpes zoster infection: two cases with MRI findings. J Neurol Sci. 2009;285(1-2):224-226. https://doi.org/10.1016/j. jns.2009.05.016

12. Rice JP. Segmental motor paralysis in herpes zoster. Clin Exp Neurol. 1984;20:129-140. PMID: 6096053

13. Esposito MB, Arrington JA, Murtaugh FR, Coleman JM, Sergay SM. MR of the spinal cord in a patient with herpes zoster. AJNR Am J Neuroradiol. 1993;14(1):203-204. PMID: 8427090

14. Cohen JI, Brunell PA, Straus SE, Krause PR. Recent advances in varicella-zoster virus infection. Ann Intern Med. 1999;130(11):922-932. https://doi. org/10.7326/0003-4819-130-11-199906010-00017

15. Miller GG, Dummer JS. Herpes simplex and varicella zoster viruses: forgotten but not gone. Am J Transplant. 2007;7(4):741-747. https://doi. org/10.1111/j.1600-6143.2006.01718.x

16. Arslan Y. UF, Ocek L., Zorlu Y. Segmental Limb Paresis Due to Zoster Plexopathy: Case Report. Turkiye Klinikleri J Neur. 2016;2(11). https://doi. org/10.5336/neuro.2016-51182

17. Mondelli M, Romano C, Passero S, Porta PD, Rossi A. Effects of acyclovir on sensory axonal neuropathy, segmental motor paresis and postherpetic neuralgia in herpes zoster patients. Eur Neurol. 1996;36(5):288292. https://doi.org/10.1159/000117274

18. Peng L, Du B, Sun L, Zhao Y, Zhang X. Short-term efficacy and safety of prednisone in herpes zoster and the effects on IL-6 and IL-10. Exp Ther Med. 2019;18(4):2893-2900. https://doi.org/10.3892/ etm.2019.7898 